\title{
Michat OBSZYŃSKI
}

Université de Gdansk

Publier de la poésie en France au XXIe siècle entretien avec Nicole Gdalia, présidente de la maison d'édition Caractères

Michał Obszyński : Bonjour, Nicole Gdalia, merci de m'accueillir ici, dans les locaux de la maison d'édition Caractères, 7 rue de l'Arbalète à Paris. Je suis très heureux de pouvoir parler avec vous aujourd'hui de la marche éditoriale en France, de la place de la poésie sur le marché littéraire, ainsi que de la littérature francophone dans votre pratique d'éditeur. Tout d'abord, je vous demanderais de présenter brièvement votre maison d'édition.

Nicole Gdalia : Je dois tout de suite préciser qu'elle n'est pas ma maison d'édition car le fondateur est Bruno Durocher, poète polonais qui, sorti du camp d'extermination de Mauthausen, est arrivé à la libération du camp en mai 1945 à Paris par la Croix Rouge polonaise. Ayant appris que toute sa famille avait été décimée dans la barbarie nazie, il décide de rester en France. Quatre années lui ont suffi pour bien maîtriser la langue française. Il décide de devenir poète français, il devient Bruno Durocher. Son premier recueil de poésie Chemin de couleur est publié en 1949 par Pierre Seghers, éditeur de poésie à la mode et important à l'époque. Durocher est aussitôt reconnu par tous les grands poètes de l'époque : Éluard, Cendrars, Char, etc., qui l'ont tous accueilli comme un des leurs. Soucieux de rentrer de plein pied dans la vie poétique et littéraire comme il l'était avant la guerre, 
Durocher rêvait de publier une revue littéraire. Ce fut dès 1950 la création de la Revue des Caractères, avec Jean Tardieu, Jean Foulain, André Frènaud, trois grandes figures de la littérature et de la poésie françaises. Très vite de gros manuscrits lui sont arrivés et ont suscité la création de la maison d'édition Caractères la même année. La maison publie de grands poètes français ou étrangers, comme Queneau, Jouve, Tzara... Des artistes sont en même temps présents : Sonia Delaunay, Jean Arp, Picasso, Krol... puisque Bruno Durocher ne concevait la poésie qu'accompagnée de l'art. Et très vite, dans les tous petits locaux qu'il avait alors, ont eu lieu des expositions, des présentations de livres de bibliophilie et de la revue Haute Feuille, toujours en compagnie d'artistes comme Picabia, Poliacov... Durocher a poursuivi ce chemin avec une très belle amplitude jusqu'en 1958 où il a été invité par le Ministre de la Culture polonais. Invitation officielle et en grand, mais dont il ne reviendra pas puisque le gouvernement communiste polonais l'arrête et le retient au pays pendant six ans. Pendant cette longue absence, sa maison, malheureusement, a été liquidée. II n'a pu revenir en France que grâce à une invitation de l'Afrique. II retournera en France via le Maroc en 1963. Durocher a donc dû tout recommencer : la revue, l'édition. Je l'ai rencontré en 1968. Le premier livre que nous avons fait ensemble, c'est Les poèmes de la révolution de mai 1968 puisque, moi-même alors étudiante, je participais activement à cette révolution universitaire de mai '68. Et nous avons uni nos vies en '68, et voilà. Au décès de Bruno Durocher en 1996, j'avais une vie universitaire de chercheur importante, mais j'ai dû, par la qualité de cette maison qui était en mouvement, me libérer de ces activités pour prendre, à plein temps, la maison d'édition et m'en occuper. J'ai eu la chance alors que les vieux poètes et amis artistes m'aient entourée et ils m'aient poussée à donner, à mon tour, ma marque à la maison d'édition Caractères. Alors, ma marque, s'il y en a une, 
c'est surtout de développer des domaines étrangers car il est devenu beaucoup plus facile d'avoir des traducteurs que vingt ans auparavant et j'ai développé la littérature étrangère : d'Asie, d'Amérique Latine et de Chine où j'ai un très bon catalogue avec deux prix Nobel chinois. II y a aussi des auteurs de langues rares comme des auteurs traduits du pachtou, comme des auteurs traduits du malayalam de I'Inde, comme des auteurs d'Afrique avec des dialectes africains. Et ce travail de découverte des domaines rares me plaît beaucoup, me passionne, je dirais...

MO : Ainsi, Caractères est une maison d'édition spécialisée en poésie, à ses débuts en poésie française, écrite en langue française et, par la suite, aussi celle du monde entier. Comment définiriez-vous la place et aussi les conditions de fonctionnement d'un éditeur indépendant spécialisé en poésie sur le marché du livre en France, à Paris ?

NG : Je précise que Bruno Durocher publiait déjà des auteurs étrangers. En 1951 il a publié des inédits de Garcia Lorca, traduits par Claude Couffon. C'est Caractères qui a fait découvrir à la France Pessoa... J'ai seulement développé le champ de la traduction, notamment avec I'Asie (Inde, Chine, Japon). Alors, je dois dire que je fais partie du marché, mais que je n'en fais pas partie non plus. C'est-à-dire que la distribution du livre en France, c'est, de plus en plus, une distribution d'environ dix titres par mois, publiés par de grandes maisons et qui traitent de scandales politiques ou érotiques ou de sujets grand public, qui font que la vente est assurée. Moi, je ne fais pas partie du tout de ce marché. Quant à la poésie, les distributeurs n'en veulent pas parce qu'elle n'est pas rentable et les libraires non plus, car elle se vend très peu. Après avoir connu trois faillites des distributeurs, après avoir perdu des livres et de l'argent, j'ai repris un système que connaissait Bruno Durocher et qui est la vente directe 
grâce à la librairie et au comptoir de ventes que nous avons. Les libraires intéressés envoient leurs coursiers. Nous utilisons aussi les réseaux sociaux, les mails qui ont beaucoup changé les relations entre éditeur et libraire. Nous leur faisons connaître nos nouveautés et nous avons des commandes ponctuelles. Nous avons aussi une boutique de ventes. Malgré tout, cela ne fait pas un chiffre d'affaire monumental. Bruno Durocher l'avait accepté, moi-même je l'accepte comme une œuvre d'art que Bruno avait commencé à sculpter, à mettre en place, et que je poursuis. Si bien que j'espère arriver à fêter en 2019 les soixante-dix ans de Caractères et, en même temps, le centenaire de la naissance de Bruno.

MO : Bénéficiez-vous d'un soutien institutionnel dans votre activité ?

NG : Oui. Alors, le système d'aide en France est un peu astreignant. Par exemple au Québec ils ont une subvention annuelle et ils la gèrent comme ils veulent. Ici c'est plus ponctuel. Chaque projet appelle le dépôt d'un dossier, l'étude de sa viabilité, de sa faisabilité. Pour les gros projets je m'y soumets, bien entendu.

MO : Juste avant notre entretien nous avons abordé le sujet des mutations dans l'industrie éditoriale en France de nos jours. Est-ce que vous pourriez présenter un peu en quoi elles consistent ? Comment influencent-elles votre activité ?

NG : Si Gutenberg a tout révolutionné avec la découverte de l'imprimerie, maintenant, ce qui révolutionne tout, c'est le numérique. Et le numérique commence à être de très bonne qualité, ce qui fait que, pour nous, c'est très intéressant parce que je peux avoir envie de faire un livre à l'ancienne, en typographie, grand format, etc. Et je peux faire un livre de série mais très beau car je suis toujours très sensible au papier que j'utilise, à la mise en pages que nous faisons. Nous travaillons beaucoup nos maquettes, 
nous travaillons beaucoup nos papiers et de ce fait je peux faire des tirages plus modestes, mais toujours très beaux et faire moins de stockage et renouveler très régulièrement notre stock au fur et à mesure qu'il s'écoule. Donc le numérique est une grande révolution. Le numérique aussi est une révolution en ce que le programme Gallica de la Bibliothèque Nationale de France s'intéresse à la réédition de nos livres du fond. Et c'est bien aussi de voir revenir à la vie, sous forme numérique, des livres très anciens publiés il y a cinquante ans...

MO : Vous avez aussi évoqué un aspect négatif, celui lié à la judiciarisation de l'édition.

NG : Oui. Dans le cadre de la commission littéraire du Syndicat de l'édition dont je suis membre, des confrères éditeurs évoquent souvent ce phénomène. II y a un essoufflement du travail de l'éditeur et il y a aussi une transformation complète de la qualité humaine, de confiance, de travail en commun qu'il y avait entre un écrivain et son éditeur. Auparavant, il y avait une fraternité des destins avec un livre, qui n'existe plus. Il y a maintenant un écrivain-homme d'affaires et un éditeur, lui aussi homme d'affaires. Et ça, ça dénature complètement le visage de l'édition. II y a un certain esprit mercantile qui prime sur justement cette passion et l'amour des lettres. Dans le passé, les écrivains étaient moins accros aux gains. Ce qui était important, c'était l'aventure de faire vivre un texte. Maintenant tout ça se dégrade. Les revendications sur les droits d'auteur, les revendications sur la durée de contrat, tout cela va tuer, je crois, malheureusement, les belles aventures littéraires.

MO : Malgré ces contraintes que vous venez de mentionner, Caractères continue cette aventure éditoriale dédiée à la poésie. Revenons à votre catalogue. Quels sont les critères de vos choix éditoriaux ? 
NG : Le critère majeur qui retenait Durocher, qui me retient aussi bien sûr, c'est la qualité. C'est la qualité d'écriture, une qualité littéraire qui s'accompagne souvent d'une qualité morale, éthique, esthétique au sens large. Et je m'efforce d'être entourée d'écrivains qui sont aussi des individus de qualité parce qu'ils partagent une certaine hauteur morale, une certaine pratique esthétique au sens du beau et du bien, vous voyez, l'esthétique au sens ancien. C'est la seule chose qui peut me permettre de tenir bon face aux difficultés économiques, sociales, etc.

MO : Au fil du temps, votre catalogue est devenu international. II englobe, entre autres, des collections dédiées aux auteurs francophones. Pouvez-vous préciser la place de ces derniers dans votre politique éditoriale ?

NG : Je n'ai pas un programme stratégique francophone. J'ai un certain nombre d'écrivains du Québec. L'Afrique m'intéresse à plus d'un titre parce qu'elle est la découverte d'une culture, d'une civilisation méconnue. Et donc, c'est avec plaisir que je l'accueille. D'ailleurs, Bruno Durocher avait déjà publié en avant-coureur des poètes africains. Je me souviens de Tchicaya U Tam'si, le Congolais dont il a publié les deux ou trois premiers livres, qui est devenu maintenant le grand poète national. Et j'ai donc ce goût de la découverte d'une culture, d'une civilisation, d'un peuple, mais pas une stratégie. Je ne cherche pas seulement la francophonie. Ce qui m'intéresse, c'est de faire connaître un univers humain, littéraire, une écriture, voilà... Les projets m'arrivent, au gré des événements, et s'ils sont de qualité je les accueille bien volontiers.

MO : En ce sens, on pourrait dire que, depuis longtemps, vous avez cette approche qui était revendiquée par les auteurs du manifeste Pour une "littérature-monde " en français, paru en 2007, et dont les signataires appelaient à la mise en avant du critère de qualité sur tout autre 
élément comme appartenance ethnique, nationale, linguistique des auteurs. Tout un programme qui serait d'ores et déjà en pratique dans votre maison d'édition.

NG : Oui, tout à fait. Ce qui compte, c'est la découverte d'un bon texte. II peut ne pas obéir du tout aux critères de sélection officielle de la codification parisienne, mais c'est un bon texte parce qu'il est authentique, parce qu'il va nous apporter des images riches, parce qu'il va nous apporter des saveurs riches et, à lui tout seul, il vaut donc, il vaut la publication, voilà... Pour moi s'y ajoute la découverte effectivement de cultures lointaines, des esthétiques d'ailleurs, de décors lointains parce que, quand je publie des nouvelles de I'Inde, j'ai tout un autre décor. Même chose quand je publie un auteur du Brésil qui parle de la rencontre des Européens avec la culture indienne du Brésil. C'est absolument passionnant de voir ces confrontations mentales, linguistiques, des rencontres de classes sociales, de cultures. Nous apprenons beaucoup de choses et c'est un enrichissement de l'humain par la littérature.

MO : Que pensez-vous donc du concept de littérature transnationale ou transculturelle qui effacerait les frontières entre les nations et les cultures, qui les ouvrirait les unes aux autres?

NG : Ça peut être très intéressant aussi. C'est-à-dire que, imaginons, comme c'est le cas de quelques-uns de nos écrivains africains par exemple, qui ont fait leurs études en France, qui sont même des enseignants dans des universités françaises et qui, peu à peu, se déconnectent d'un terroir et obligatoirement leur terroir devient celui dans lequel ils vivent. Automatiquement leur littérature va se transplanter, va devenir un greffon de la littérature française et l'écrivain devient un écrivain français. Voilà.

MO : Pour terminer notre entretien, pourriez-vous présenter les projets qui vous attendent? 
NG : Les projets littéraires sont très nombreux. J'ai travaillé ce matin sur un pays d'Asie, pour un projet de livre qui va inclure poésie, art, histoire. II y a aussi un jeune poète qui m'apporte sa poésie chantée. J'ai déjà publié deux livres de lui et je l'ai suivi parce qu'il a beaucoup de talent et lui m'est aussi très fidèle. Après quelques années où il ne m'a pas donné un texte pur et où il a fait beaucoup de concerts de poésie chantée, il est revenu vers moi pour publier les textes de ses poèmes mis en musique. Comme il est très authentique dans sa poésie et que c'est un bon poète, j'ai accepté et nous avons conversé tout à l'heure au téléphone. J'ai des textes d'Amérique latine, de Suède, d'Israël... En 2018 est prévue une année d'échanges culturels France-Israël.

MO : Vous êtes aussi en train de terminer l'édition des œuvres complètes de Bruno Durocher.

NG : Oui. Et c'est le quatrième volume qui est presque achevé actuellement. On est dans les dernières relectures, le choix du papier, etc. Et je voudrais, à l'issue de la publication de ce quatrième volume et donc de l'œuvre complète, prévoir quelques manifestations, lectures, soirées de poésie. Voilà ce que je pourrais faire autour de l'œuvre de Durocher et puis aussi je voudrais m'occuper de mon œuvre... Je suis en préparation de publication d'un poème dramatique avec la contribution de trois artistes et d'un compositeur...

MO : Je vous souhaite donc bon courage pour la suite de tous ces projets et je vous remercie pour cet entretien.

NG : Je vous remercie infiniment et au plaisir.

Entretien réalisé le 15 décembre 2016 dans le cadre d'une bourse de recherche postdoctorale financée par le Centre National de la Science en accord avec la décision n० DEC2015/16/S/HS2/00124. 


\section{abstract}

Publishing poetry in France in the 21st century interview with Nicole Gdalia, head of the publishing house Caractères

The following interview aims at examining the role of an independent Parisian publishing house on the French book market. Economic and institutional circumstances surrounding smaller French publishing houses are illustrated by the example of Caractères publishing house, which specializes in poetry. At the same time, the interview raises the issue of francophone writing in the publishing strategy of Caractères in the light of a debate on the diversity of francophone literatures and littératuremonde concept.

\section{keywords}

French Book market, independent publisher, francophone poetry, Éditions Caractères

\section{mots clés}

marché du livre en France, édition francophone, maisons d'édition indépendantes, Éditions Caractères

\section{michał obszyński}

Michał Obszyński est actuellement chercheur à I'Université de Gdansk. II concentre ses recherches sur les aspects socio-politiques de la production littéraire francophone des Amériques et s'intéresse plus particulièrement aux stratégies éditoriales à l'œuvre au sein de la francophonie littéraire. 